\title{
15 Schule für Betreuung und berufliche Weiterbildung Jugendlicher und junger Erwachsener mit einer schizophrenen Erkrankung
}

Schizophrene Patienten leiden unter vielfachen kognitiven Leistungseinbußen, Aufmerksamkeits- und Konzentrationsstörungen sowie Beeinträchtigungen des planenden, zielorientierten und situationsrelevanten Denkens und Handelns. Diese Schwierigkeiten hängen u.a. mit der schizophrenietypischen Unfähigkeit zusammen, Problemlösestrategien entsprechend den speziellen aufgabenrelevanten Anforderungen anzupassen, eingefahrene Denk- und Handlungsschemata („kognitive Rillen“) flexibel zu verbessern und fokussierte Strategiewechsel vorzunehmen (vgl. Kap. 5.8). Daraus resultieren Inflexibilität einerseits und Hyperimpulsivität andererseits, die Denken und Handeln schizophrener Jugendlicher typischerweise kennzeichnen.

Aus diesen Gründen und aufgrund oftmals langer Fehlzeiten in der Schule können die betroffenen Jugendlichen oft nicht in der Regelzeit einen ihnen angemessenen Schulabschluss erreichen. Deshalb hat die Prof. Christian Eggers-Stiftung in Zusammenarbeit mit der VHS in Essen einen zweijährigen Lehrgang installiert, der diesen jungen Menschen die Möglichkeit eröffnet, in diesem Zeitraum die Fachoberschulreife zu erreichen. Daran nehmen jeweils 15 Jugendliche und junge Erwachsene teil, die infolge ihrer psychischen Erkrankung bisher nicht zu einem Schulabschluss gelangt sind. Der Kurs wurde im Jahr 2006 eingerichtet, so dass bisher zwei Lehrgänge mit insgesamt 25 Teilnehmern erfolgreich abgeschlossen werden konnten - zu den Ergebnissen siehe nachfolgenden Bericht von H. Reintanz-Vanselow. Trotz erheblicher Beeinträchtigungen - die Teilnehmer/-innen waren zum Teil chronisch-psychotisch, standen unter dem Einfluss kommentierender, entwertender imperativer Stim- 
men („mach dich weg“, „du ..., du bist der letzte Dreck“, „bring dich um ...“) und litten unter multiplen Wahnvorstellungen (v.a. unter Beziehungs-, Bedeutungs-, Beeinträchtigungs- und Verfolgungsideen) und nahmen z.T. hochdosiert Antipsychotika. Unter diesen Bedingungen innerhalb von 2 Jahren die Fachoberschulreife $z u$ erreichen, ist eine nicht hoch genug einzuschätzende moralische Leistung!

Hinzu kommt, dass die beschriebenen kognitiven Leistungseinschränkungen oft schon in der prämorbiden Periode, vor Krankheitsbeginn, bestehen, aber leider nicht selten unbemerkt bleiben oder als Lernstörung, Faulheit oder ADS-Symptomatik (attention deficit syndrome) fehlinterpretiert werden (s. auch Kap. 5.8). Eine rezente Langzeitstudie hat nun ergeben, dass die neurokognitiven Defizite bei Kindern und Jugendlichen mit einer Frühschizophrenie (EOS = early onset schizophrenia) im Langzeitverlauf stabil bleiben oder sich sogar verschlechtern (Frangou et al. 20o8, Øie et al. 2010). Im Vergleich zu einer ADHD-Gruppe (ADHD = attention deficiency hyperactivity disorder) und einer psychiatrisch unauffälligen Kontrollgruppe wurden bei 15 schizophrenen Jugendlichen nach 13-jährigem Psychoseverlauf eine signifikante Verschlechterung oder ein Stillstand der neurokognitiven Entwicklung festgestellt, v.a. im Bereich des verbalen Gedächtnisses, der Aufmerksamkeitsfunktionen und der Problemlösegeschwindigkeit (Øie et al. 2010). Diese drei Leistungsbereiche korrelieren in starkem Maße mit dem Krankheitsausgang und der sozialen Funktionsfähigkeit der Betroffenen (Green et al. 2004). Die neurokognitiven Leistungseinbußen dürften mit den in Kapitel 12.3-12.5 beschriebenen neuroanatomischen Veränderungen zusammenhängen und typisch gerade für die Frühschizophrenie sein (vgl. Kap. 12.4 und 12.5). Zusätzlich wirkt sich die neuroleptische Behandlung ungünstig auf die kognitive Leistungsfähigkeit aus.

Es ist schwierig zu erfahren bzw. zu verstehen, wie ein schizophrenes Individuum denkt, antizipiert, plant und ein ihn betreffendes Problem bewältigt, und wie ihm dies in den verschiedenen Stadien seiner Psychose gelingt oder eben auch misslingt. Auch das Wissen über die neurobiologischen Bedingungen, die ein erfolgreiches Lernen bei schizophrenen Patienten ermöglicht, liegen noch im Dunkeln (Holcomb $u$. Murray 2010). Um einer Klärung dieser Probleme näher zu kommen, sind Studien nötig, die verhaltensphysiologische, magnetresonanz-tomographische, PET-, SPECTund DTI-Untersuchungen miteinander verbinden (Holcomb u. Murray 2010).

Eine gute schulische und berufliche Ausbildung stellt aber einen wichtigen, wenn nicht entscheidenden protektiven Faktor für die weitere Krankheitsentwicklung und für das Erreichen eines befriedigenden Arbeitsplatzes dar (Gold et al. 2006, McGurk u. Mueser 2004, Kooyman et al. 2007). Die Verhinderung von Arbeitslosigkeit hat erhebliche Auswirkungen auf den Selbstwert und die Lebensqualität eines jungen Menschen. Ein negativer Selbstwert macht anfällig für Hoffnungslosigkeit, Selbstdiskriminierung, Verzweiflung, depressive Symptome und eine daraus resultierende erhöhte Suizidalität. Außerdem wird dadurch die Rückfallgefahr erhöht.

Verlaufsuntersuchungen zeigen, dass ca. 6o\% der im Kindes- oder Jugendalter Erkrankten keiner beruflichen Tätigkeit nachgehen und auf finanzielle Unterstützung aus sozialstaatlichen Fördermitteln angewiesen sind. Die daraus folgende Perspektivlosigkeit befördert die Chronifizierung der psychotischen Erkrankungen.

Deshalb haben wir in Essen in Zusammenarbeit der Prof. Eggers-Stiftung und der VHS, unterstützt durch Prof. Dres. h.c. Bertold Beitz, ein Förderkonzept für Jugendliche und junge Erwachsene entwickelt, welches ihnen den Einstieg in das Berufsleben 
erleichtern soll. Das Projekt hat zunächst eine Laufzeit von 4 Jahren (vom 01.10.2008 bis 30.09.2012).

Das Konzept dieses Projekts setzt sich aus folgenden Elementen zusammen:

- Stärkung der persönlichen und sozialen Kompetenzen

- Professionelles Bewerbungstraining

- Präsentationstraining

- Kommunikationstraining

- Image- und Typberatung

- EDV-Schulung

- Berufspraktika

- Theater- und kunstpädagogische Projekte

- Psychologische Begleitung

- Öffentlichkeitsarbeit

- Lehrerfortbildungen zum Umgang mit psychisch erkrankten jungen Menschen

Bisherige Erfahrungen belegen, dass die jungen Leute aufgrund ihrer langjährigen Erkrankung meistens nur noch sehr wenig Selbstvertrauen haben und im Alltag häufig Einschränkungen wie Ängste, Stimmungsschwankungen oder Konzentrationsstörungen überwinden müssen und außerdem mit gesellschaftlichen Vorbehalten konfrontiert sind. Das hat nicht selten Motivationsprobleme zur Folge! Unter diesen Voraussetzungen haben die Betroffenen Schwierigkeiten, in Bewerbungsgesprächen das Vertrauen potentieller Arbeitgeber zu gewinnen und in Stellenbesetzungsprozessen erfolgreich zu sein. Sie benötigen Selbstvertrauen, Mut und kommunikative Kompetenz, um in der Arbeitswelt ihre besonderen Stärken trotz ihrer schwierigen Biographie (z.B. krankheitsbedingte Lücken im Lebenslauf) zum Ausdruck bringen zu können.

Deshalb wird den Betroffenen durch ein Bewerbungstraining das Erlernen von Präsentationstechniken und das Erstellen von aussagekräftigen Bewerbungsunterlagen vermittelt, wodurch der Weg für eine erfolgreiche Bewerbung geebnet wird. Dieser Baustein wird durch zielgruppenspezifische Angebote ergänzt, die die seelischen Entwicklungsstörungen berücksichtigen. Dazu zählen theater- und kunstpädagogische Projekte, um Mut und Selbstvertrauen für das Leben außerhalb eines geschützten Rahmens aufzubauen. Ein Psychologe und die Projektleitung stehen den Teilnehmenden für die psychologische Beratung und die psychosoziale Begleitung zur Verfügung. Betriebspraktika fördern eine berufliche Orientierung der Teilnehmenden und bereiten sie allmählich auf den Arbeitsalltag vor.

Von besonderer Bedeutung ist das Kommunikationstraining mit dem Ziel der Ermöglichung, eigene Interessen vertreten und interpersonelle Konflikte kompetent meistern zu können. Dies ist besonders wichtig, da psychotische oder psychose-ähnliche Störungen häufig damit verbunden sind, dass erkrankte Menschen sich leichter angegriffen, verletzt oder bedroht fühlen. Sie müssen daher lernen, in solchen Situationen aus eigener Initiative Probleme in respektvoller und angemessener Weise anzusprechen und kritische Situationen im Vorfeld zu entschärfen.

In einem theaterpädagogischen Projekt wird die Befähigung zur Arbeit im Team geübt. Die Leistungen des Einzelnen fügen sich zu einem gemeinsamen Ergebnis zusammen. Da Menschen mit psychotischen oder psychose-ähnlichen Erkrankungen an oft gravierenden Konzentrationsstörungen leiden, ist es für sie eine große Heraus- 
15 Schule für Betreuung und berufliche Weiterbildung Jugendlicher und junger Erwachsener mit einer schizophrenen Erkrankung

forderung, Texte zu lesen und zu erfassen. Zudem fällt es ihnen schwer, Wichtiges von Unwichtigem zu unterscheiden. Im Theaterprojekt werden Konzentration und Merkfähigkeit trainiert. Die jungen Erwachsenen nähern sich Texten auf eine neue, spielerische Weise. Auch hier fördert der kreative Prozess die Identitätsbildung.

\title{
15.1 An gute Noten muss ich mich erst gewöhnen - Erfahrungsbericht aus einem Modellprojekt für psychisch erkrankte Jugendliche und junge Erwachsene
}

\author{
Von Heike Reintanz-Vanselow
}

\section{Einleitung}

„An gute Noten muss ich mich erst gewöhnen“, so lautet die erstaunte Reaktion eines jungen Mannes, der in der Volkeshochschule den mittleren Bildungsabschluss nachholt und ein „gut" unter seiner Deutschklausur entdeckt. Solche schulischen Erfolgserlebnisse waren für ihn lange Zeit unvorstellbar. Er lebt mit der Diagnose „schizophrene Psychose" und hat Phasen extremer Beeinträchtigung seiner kognitiven Fähigkeiten sowie der Leistungs- und Konzentrationsfähigkeit durchlebt. So ist es nicht erstaunlich, dass in seiner Reaktion auch Skepsis mitschwingt, ob sich diese gute Leistungen dauerhaft realisieren lassen würden. Seine Angst vor Misserfolgen ist groß und lässt jede Klassenarbeit und jedes Referat zu einer riesigen Hürde anwachsen. Aber mit jeder erfolgreich gemeisterten Aufgabe wachsen auch sein Selbstbewusstsein und der Mut, sich neuen Herausforderungen zu stellen.

In einem Projekt der Eggers-Stiftung in Zusammenarbeit mit der Volkshochschule Essen erhalten 15 psychotisch oder anderweitig psychisch erkrankte junge Erwachsene die Möglichkeit, innerhalb von zwei Jahren den mittleren Bildungsabschluss zu erwerben. Alle Teilnehmenden haben ihre Pflichtschulzeit beendet, blieben aber aufgrund ihrer psychischen Erkrankungen ohne Schulabschluss oder erreichten einen Hauptschulabschluss mit sehr schwachen Noten. Ein qualifizierter Schulabschluss ist aber eine notwendige Grundvoraussetzung für einen Einstieg in das Berufsleben, der eine wichtige Bedeutung für ein späteres selbstständiges und erfülltes Leben hat. Insofern soll der Kurs den jungen Menschen die Chance geben, einen qualifizierten Schulabschluss zu erreichen und ihre kognitiven und sozialen Kompetenzen weiterzuentwickeln, um die Voraussetzungen für ihre Teilnahme am Erwerbsleben zu schaffen. Die zentrale Bedeutung der Berufstätigkeit für Menschen mit psychotischen Erkrankungen heben auch Michaela Amering und Margit Schmolke $(2007)^{7}$ hervor.

„Die Teilnahme am Berufsleben spielt eine entscheidende Rolle für die Bewältigung von Alltag und Erkrankungsrisiko. Sowohl aus therapeutischer Sicht als auch aus Sicht der Patienten sind Hilfsangebote, die den Einstieg oder Wiedereinstieg in Ausbildungs- oder Arbeitssituationen ermöglichen, ein wesentlicher Schritt zu Lebensqualität und zur Verhinderung von Rückfällen und Chronifizierung." (Amering und Schmolke 2007, S. 53) 
Der nachfolgende Bericht fasst die Erfahrungen nach den ersten vier Jahren im Projekt zusammen.

Nach der Darstellung der Rahmenbedingungen wird das pädagogische Konzept ausführlich erläutert. Die bisherigen Resultate des andauernden Modellprojektes werden im abschließenden Fazit zusammengefasst.

\section{Individuelle Förderung durch angepasste Rahmenbedingungen}

Das Modellprojekt ist für junge Menschen zwischen 18 und 25 Jahren, die an einer schizophrenen Psychose leiden, vorgesehen. Durch Eingangstests wird zunächst eine Lernbehinderung ausgeschlossen. Die Teilnehmenden haben z.T. die Haupt- und Realschule, z.T. das Gymnasium besucht. Aufgrund der Erkrankungen weisen viele Teilnehmende gravierende Defizite der kognitiven Leistungsfähigkeit auf. Weiterhin ist häufig krankheitsbedingt ihre Motivations- und Konzentrationsfähigkeit eingeschränkt und sie haben Schwierigkeiten, Wesentliches und Unwesentliches zu unterscheiden. Einigen von ihnen fällt es sehr schwer, zielgerichtet zu denken und ihr Handeln zu planen. Manche Teilnehmende sind anderen gegenüber sehr misstrauisch und entwickeln schnell Beziehungsideen. Die Teilnehmerzahl im Kurs ist auf höchstens 15 Personen begrenzt, um eine individuelle Förderung gewährleisten zu können, aber auch um eine Reizüberflutung der Teilnehmenden durch zu viele Menschen zu vermeiden. Die Sitzordnung ist so eingerichtet, dass alle Teilnehmenden sich anschauen können und nicht mit dem Rücken zueinander sitzen. Dies fördert einerseits die Kommunikation in der Gruppe und unterstützt den Aufbau von Vertrauen zueinander.

Der Professor-Eggers-Lehrgang ist auf die Bedürfnisse junger Erwachsener mit psychischen Erkrankungen zugeschnitten und bietet folgende Rahmenbedingungen:

- eine ruhige, reizarme Umgebung

- eine überschaubare Cruppe mit 15 Teilnehmenden

- psychosoziale Betreuung durch einen Psychologen und eine Lehrerin

- integrierte therapeutische Beratung

- koordiniertes Vorgehen des pädagogischen Teams

- individuelle Förderung mit Rücksicht auf die Erkrankungen der Teilnehmenden

- Möglichkeit eines gestuften Einstiegs (allmählich ansteigende Zahl der Unterrichtsstunden)

\section{Schulische Erfolge durch ein bedarfsgerechtes pädagogisches Konzept}

Aus den Erfahrungen im Professor Eggers Lehrgang entwickelte das pädagogische Team ein Konzept, das auf die Bedürfnisse der Teilnehmenden abgestimmt ist und sich immer wieder den aktuellen Anforderungen anpasst. Im Folgenden soll dieses pädagogische Konzept erläutert werden.

\section{Wertschätzung}

Die pädagogische Arbeit im Kurs basiert grundsätzlich auf der Wertschätzung für die Teilnehmenden. Sie können sich zu jeder Zeit akzeptiert und Ernst genommen fühlen. Dies ist eine Grundvoraussetzung, um junge Menschen zu erreichen, die im 
Laufe ihrer Jugend häufig Unverständnis, Skepsis und Ablehnung erfahren haben. Viele berichten, dass sie in ihrer Schulzeit Misserfolge, Ausgrenzung und Stigmatisierung erlebt haben. Daher schaffen wir zunächst eine vertrauensvolle Atmosphäre. Die Teilnehmenden spüren, dass wir sie schätzen und ihre besonderen Begabungen wie Kreativität oder soziale Kompetenz erkennen. Auch ist es sehr wichtig, die Teilnehmenden spüren zu lassen, dass uns ihr Wohlergehen wichtig ist und am Herzen liegt. Das folgende Beispiel veranschaulicht die entscheidende Bedeutung dieser vertrauensvollen, persönlichen Bindung.

Eine Teilnehmerin des Lehrgangs hatte über längere Zeit immer wieder unentschuldigt gefehlt und war schließlich gar nicht mehr zum Unterricht erschienen. Die Lehrgansleiterin hatte sie immer wieder angerufen und sie hatte dann versprochen, am nächsten Tag wieder zu kommen, was aber nicht geschah. In den Telefonaten berichtete die Teilnehmerin, dass sie sich antriebslos und verängstigt fühle, dazu an Übelkeit litte und es einfach nicht schaffe „vom Sofa aufzustehen“. Sie erhielt daraufhin die Auflage, an einem persönlichen Gespräch mit dem begleitenden Therapeuten und der Lehrgangsleitung teilzunehmen.

Zu Beginn des Gesprächs brachte die Lehrgangsleiterin ihre Sorge um die Teilnehmerin zum Ausdruck, denn es war sehr deutlich, dass es ihr schlecht ging. Auch teilten wir ihr mit, dass wir uns sehr wünschten, sie weiterhin im Lehrgang zu behalten und weiter mit ihr zu arbeiten. Das Gespräch habe das Ziel, gemeinsam herauszufinden, was sie am Schulbesuch hindere und Strategien zu erarbeiten, wie sie dennoch zum Unterricht kommen könne. Wir fragten auch, in welcher Weise wir sie unterstützen könnten. Es wurde schnell deutlich, dass die junge Frau durch ihre gesamte Lebenssituation überfordert war. Sie lebte nach zwei akuten Psychosen, die mit stationärer Behandlung verbunden waren, allein in einer eigenen Wohnung und hatte keinerlei Betreuung, weder durch Angehörige noch durch Sozialarbeiter. Sie hatte versucht alles „alleine zu schaffen“, war aber schon damit überfordert, morgens aufzustehen, zur Schule zu gehen und insbesondere auch damit, für die Schule am Nachmittag zu arbeiten. Sie trank häufig Alkohol, um sich abzulenken und konsumierte gelegentlich Marihuana. Eine Suchtberatung hatte sie bereits aufgesucht, jedoch nur mit zeitweiligem Erfolg. Sie schämte sich für ihre Unfähigkeit, den Alltag zu bewältigen und dafür, dass sie es nicht schaffte, zur Schule zu gehen. Ihre Scham lähmte sie und hielt sie davon ab, nach einigen Fehltagen wieder zur Schule zu gehen. Auch war sie nicht in der Lage zuhause zu arbeiten und wäre daher ohne Hausaufgaben zur Schule gekommen, was sie als persönliches Versagen empfand. Dies hat teufelskreisartig ihre Selbstscham verstärkt nach dem Motto „Ich schäme mich, bin nichts wert, also resigniere ich, raffe mich nicht auf, fliehe in Alkohol und Drogen, verleugne die Realität, erlebe mich als Versagerin, was mein Selbstvertrauen wiederum verstärkt" etc.

In diesem Gespräch versicherten wir der jungen Frau, dass wir sie sehr schätzen und ihr Fernbleiben nicht als Versagen, sondern als Resultat ihrer Überforderung verstehen. Wir schlugen vor, gemeinsam zu überlegen, wie wir die Anforderungen so gestalten könnten, dass sie es wieder wagen würde, zur Schule zu kommen. Da sie Unterstützung in alltäglichen Belangen benötigte, empfahlen wir ihr, sich um eine ambulante Betreuung zu bemühen und vermittelten den Kontakt. Für die fehlenden Arbeiten und Hausaufgaben erarbeiteten wir einen Plan mit Abgabeterminen, die für sie realistisch waren. Sie versprach sich zu melden, wenn sie sich überfordert fühlen würde.

Am nächsten Tag erschien sie wieder zur Schule, allerdings mit einer Verspätung von zwei Stunden. Die Lehrerin lobte sie dafür, dass sie gekommen war und ermutigte sie, weiterhin 
zu kommen, auch wenn sie sich zunächst verspäten würde. Im Laufe der nächsten Wochen nahm die Zahl der Verspätungen ab. Sie fehlte noch hin und wieder, besonders dann, wenn die Anforderungen stiegen und sie sich überfordert fühlte. Es gelang ihr, mit den Lehrern das Gespräch zu suchen und Vereinbarungen zu treffen, wie sie Aufgaben nachholen könnte. Mit jeder Woche, die sie den Unterricht regelmäßig besuchte, wuchsen ihr Selbstvertrauen und die Zuversicht, den Schulabschluss tatsächlich zu erreichen. Die Fehlzeiten reduzierten sich und die Chancen auf einen erfolgreichen Abschluss des Lehrgangs haben sich verbessert.

Einige Tage nach dem Interventionsgespräch erklärte die Teilnehmerin der Lehrgangsleiterin, warum sie nun wieder zum Unterricht kam und die Hilfe angenommen habe. Es sei für sie ganz entscheidend gewesen, dass sie unser Interesse und unsere Empathie gespürt habe. Sie habe gemerkt, dass es uns wichtig war, was aus ihr werde und ob sie zur Schule komme. Das habe sie dazu bewogen, es noch einmal zu versuchen. Wenn wir jedoch Drohungen und Abmahnungen ausgesprochen hätten, hätte sie das nicht überzeugen können, sondern vielmehr dazu geführt, dass sie sich endgültig zurückgezogen hätte.

Diese Geschichte zeigt, dass die Teilnehmer unsere Empathie und unsere Fürsorge in besonderem Maße benötigen. In diesem Sinne übernehmen Lehrerin und Therapeut die Funktion von „guten und liebevollen Eltern“, die ihr Kind annehmen und unterstützen, auch wenn es nicht den Erwartungen der Eltern entspricht oder die gewünschten Leistungen erbringen kann.

\section{Kompetenzorientierung}

Das Selbstbild der Teilnehmenden ist durch Misserfolgserlebnisse sehr fragil und so besteht eine weitere wichtige Aufgabe darin, durch Lob und Anerkennung die Teilnehmenden zu ermutigen, ihre eigenen Fähigkeiten zunächst zu erkennen und dann auf diese zu vertrauen. Auf dieser Grundlage beginnen die jungen Menschen, sich realistische Ziele zu setzen, auch wenn damit das Risiko eines eventuellen Misserfolgs verbunden ist. Diese Arbeit erfordert viel Empathie und Geduld, denn die individuellen Hindernisse sind teilweise sehr groß und wenig vorhersehbar. Es gilt, mit und von den Teilnehmenden zu lernen und gemeinsam Strategien zu entwickeln.

Das Beispiel einer Teilnehmerin mit Prüfungsangst zeigt, wie sich dieser Anspruch im Schulalltag umsetzen lässt.

Eine junge Frau besuchte seit etwa einem Jahr den Schulabschlusslehrgang und ihre mündlichen und schriftlichen Leistungen waren fast immer gut oder sogar sehr gut. Dennoch plagten sie vor jeder Klausur starke Ängste. Sie war fest davon überzeugt, die Aufgabe niemals bewältigen zu können. In einer Prüfungssituation las sie die Aufgabenstellungen und begann mit der Arbeit. Allerdings brach sie nach den ersten beiden Sätzen ab, raufte sich die Haare, wurde sehr unruhig und begann zu weinen. Auf Nachfrage der Lehrerin erklärte sie, dass sie gar nichts könne und es niemals schaffen werde. Die Lehrerin erinnerte daran, dass die Teilnehmerin bisher alle Arbeiten erfolgreich beendet habe und ja die Erfahrung gemacht habe, dass sie ihre Angst überwinden könne. Sie schlug der Teilnehmerin vor, eine kurze Pause einzulegen und es dann noch einmal zu versuchen. Dabei solle sie mit der Aufgabe beginnen, die sie am leichtesten bewältigen könnte. Nach einer Pause begann die Teilnehmerin erneut, fühlte sich aber immer noch überfordert. Die Lehrerin schlug ihr vor, sich auf eine der vier Aufgaben zu beschränken, die sie erfahrungsgemäß gut bewältigen konnte. Sie 
würde die Chance erhalten, die Arbeit zu einem späteren Zeitpunkt zu wiederholen, sollte aber dennoch versuchen, die eine Aufgabe zu bearbeiten. Dies führte zu einer Entlastung der Teilnehmerin. Sie bearbeitete schließlich die Aufgabe und überwand allmählich die Blockade. Nun versuchte sie doch noch, auch die anderen Aufgaben zu lösen und schaffte es, die Klausur fast vollständig zu bearbeiten. Bei der Abgabe lobte die Lehrerin sie ausdrücklich und hob die bemerkenswerte Leistung der Teilnehmerin hervor, die nicht nur ihre Ängste erfolgreich überwunden, sondern auch noch ihre Klausur fast vollständig bearbeitet hatte.

Der Erfolg dieses Vorgehens beruht auf einer entlastenden Intervention. Einerseits wird der Leistungsdruck reduziert; die Teilnehmerin erhält die Möglichkeit, die Klausur nachzuholen, und sie darf sich auf eine Aufgabe beschränken. Andererseits orientiert sich das Vorgehen an den Fähigkeiten der Teilnehmerin, die selbst entscheiden kann, welche Aufgabe sie bearbeitet. Da sie mit einer Aufgabe beginnt, die sie gut beherrscht und erfolgreich bearbeiten kann, beginnt sie wieder Vertrauen in ihre Fähigkeiten aufzubauen und bearbeitet schließlich auch weitere Aufgaben.

\section{Kreativität und Lösungsorientierung}

Da die Lernenden sehr unterschiedliche Hürden beim Lernen überwinden müssen, ist der Ideenreichtum der Lehrenden gefordert. Ein Beispiel aus dem Deutschunterricht soll dies veranschaulichen.

Für viele Menschen, die an einer Psychose leiden, ist es nicht leicht, Wesentliches von Unwesentlichem zu unterscheiden und Informationen nach ihrer Relevanz zu ordnen. Es ist daher eine große Herausforderung für viele unserer Teilnehmenden, die Inhaltsangabe eines Textes zu erstellen. Die Methode, zunächst die wichtigsten Informationen des Textes zu unterstreichen ist wenig hilfreich, weil sie die Lernenden in diesem Falle überfordert. Sie versuchen dann verzweifelt, im Text nach „wichtigen“ und „unwichtigen“ Informationen zu suchen und am Ende ist beinahe alles unterstrichen. Es ist daher sehr sinnvoll, die Teilnehmenden mit für sie lösbaren Aufgaben auf das Verfassen der Inhaltsangabe vorzubereiten, um solche Frustrationen zu vermeiden.

Eine Möglichkeit besteht darin, zunächst kurze Nachrichten aus der Zeitung im Unterricht zu besprechen, die dem Leser in knapper Form die zentralen Informationen zusammenfassen, etwa über kleinere Diebstähle, Einbrüche usw. Die Teilnehmer erkennen schnell, dass hier nur die Fragen wer?, wo?, wann?, was?, wie? und warum? beantwortet werden und keinerlei ausschmückende Details enthalten sind. Im nächsten Schritt erhalten die Teilnehmer dann die Aufgabe, eine solche Meldung in einen Kurzkrimi zu verwandeln, indem sie die „nackten Informationen" ausschmücken, die handeInden Personen und den Ort der Handlung näher beschreiben. Die Teilnehmenden erarbeiten Methoden, wie sie ihrem Text Spannung erzeugen können. Sie ergänzen Dialoge und fügen Gedankengänge der handelnden Personen ein. Für diese Arbeit können die Lernenden ihre oft sehr ausgeprägte Fantasie und Kreativität gut einsetzen und damit ihrer besonderen Begabungen einsetzen.

Im nächsten Schritt lernen die Teilnehmenden, aus einem Text alle ausschmückenden Elemente zu streichen und auf diese Weise die zentralen Informationen herauszuarbeiten. Es fällt ihnen so viel leichter zu verstehen, welche Informationen für das Verständnis des Textes benötigt werden und welche Textpassagen der näheren Erläuterung und Beschreibung dienen. Auf diese Weise werden die Teilnehmenden schrittweise mit Aufgaben, die sie be- 
wältigen können, an eine neue Aufgabe herangeführt. Sie freuen sich über ihre Erfolge und fühlen sich gefordert aber niemals überfordert, denn niemand verlangt, dass sie „Wichtiges“ von „Unwichtigem“ unterscheiden sollen.

Überforderung kann man im Unterricht sehr gut erkennen, wenn Teilnehmer sich plötzlich zurückziehen, sich nicht mehr beteiligen oder auch auf ganz andere Tätigkeiten ausweichen.

Eine Teilnehmerin begann einmal, in ihr Tagebuch zu schreiben, obwohl Sie die Aufgabe hatte, nach erfolgter Stoffsammlung eine Gliederung für eine Erörterung zu erstellen. Auf Nachfrage der Lehrerin, warum Sie nicht weiterarbeite sagte sie: „ich fühle mich heute gar nicht gut und muss vielleicht gleich gehen". Die Lehrerin bot ihre Hilfe bei der Arbeit an und erklärte noch einmal, dass es hier darum gehe, die für den Leser der Erörterung überzeugendsten Argumente aufzulisten und in eine sinnvolle Reihenfolge zu bringen. Dabei sollte Sie die Argumente nach ihrer Überzeugungskraft ordnen und mit dem „wichtigsten“ Argument enden. Daraufhin sagte die Teilnehmerin: „|a, das ist aber gar nicht so einfach, wenn man eine Psychose hat!“.

Schnell wurde klar, dass sie generell Schwierigkeiten hatte, zwischen Wesentlichem und Unwesentlichen zu unterscheiden. Andere Teilnehmer räumten ebenfalls ein, dass sie die Aufgabe nicht ohne Hilfe lösen könnten. Die Lehrerin schlug vor, ein gemeinsames Brainstorming zum Thema durchzuführen. Alle Gedanken zum Thema sollten gesammelt werden. So fanden sich an der Tafel schließlich auch Punkte wie „ich finde das Thema blöd“ oder "Klassenarbeit" und "Raucherpause“ wieder, die keinerlei Bezug zum Thema hatten. Gemeinsam gelang es schnell, alle Punkte zu streichen, die für die Erörterung irrelevant waren. Bei allen relevanten Punkten überlegte die Gruppe gemeinsam, wie überzeugend und aussagekräftig sie sind. Schließlich gelang es allen, eine Liste der Argumente zu erstellen und diese nach ihrer Relevanz für das Thema zu ordnen.

Aus diesem Beispiel wird deutlich, dass die Verweigerung der Mitarbeit oft vielschichtige Ursachen haben kann und nach unserer Erfahrung meist auf eine Überforderung hindeutet. Es ist wichtig, nicht vorschnell zu urteilen, sondern lösungsorientiert zu arbeiten. Von den Lehrenden wird in hohem Maße Kreativität und Methodenvielfalt gefordert, um individuelle Lösungsstrategien zu entwickeln.

\section{Klare Regeln und Strukturen}

Die Grundlage für solche individuellen Konzepte bilden feste Regeln und Strukturen in der Gruppe. Hierzu gehören insbesondere das Einhalten von in der Gruppe vereinbarten Gesprächs- und Verhaltensregeln, pünktliches und regelmäßiges Erscheinen sowie die sofortige Benachrichtigung der Lehrkraft im Krankheitsfall und das Einreichen von Attesten bei Fehlzeiten.

\section{Binnendifferenzierung}

Da die Gruppe hinsichtlich der schulischen Vorbildung nicht homogen ist, werden die verschiedenen Lernniveaus durch differenzierte Aufgaben in den Unterricht integriert. Während etwa in Mathematik eine Cruppe den aktuellen Stoff noch erarbeitet, kann eine andere Cruppe bereits weiterführende Aufgaben lösen. Im Biologie- 
unterricht können Teilnehmende, die den Stoff bereits gut beherrschen, ein neues Thema recherchieren und als Kurzreferat in den Unterricht einbringen. In ähnlicher Weise ist in allen Fächern Binnendifferenzierung durch abgestufte Arbeitsmaterialien und Aufgabenstellungen möglich. Fortgeschrittene Teilnehmer können als Tutoren andere bei ihrer Arbeit unterstützen und so eine wichtige Funktion in der Gruppe übernehmen. Das Lerntempo in der Gruppe ist sehr unterschiedlich, doch durch differenzierte Aufgabenstellungen ermöglichen wir es den Teilnehmenden, im Rahmen der Zeitvorgaben ihr eigenes Tempo zu finden

\section{Methodenvielfalt und Handlungsorientierung}

Die Konzentrationsfähigkeit ist bei nahezu allen Teilnehmenden stark eingeschränkt. Häufig schweifen die Gedanken vom Thema des Unterrichts ab. Einige Teilnehmende hören auch Stimmen, die sie ablenken. Insgesamt ermüden sie sehr schnell und fühlen sich sowohl durch längere Wortbeiträge oder Vorträge als auch durch intensive Gruppendiskussionen rasch überfordert. Wir verzichten daher weitgehend auf lehrerzentrierten Unterricht und vermeiden längere Lehrervorträge. Nach einer kurzen Einführung wechseln sich Phasen der Einzel-, Cruppen- oder Partnerarbeit ab. Für den Unterricht stehen zwei miteinander verbundene Räume zur Verfügung. Teilnehmende, die mehr Ruhe brauchen, können sich für die Phasen der Einzelarbeit in den zweiten Raum zurückziehen oder eine „Auszeit“ nehmen, wenn sie sich überfordert fühlen. Mit dieser Möglichkeit, selbstbestimmt Arbeitspausen einzulegen, gehen die Lernenden sehr gezielt und verantwortungsvoll um.

Im handlungsorientierten Unterricht steht das eigenständige Lösen von Aufgaben im Mittelpunkt. Mithilfe von Texten, Medien oder in Biologie von Experimenten, erarbeiten die Teilnehmenden Lösungen, die in Form von Wandzeitungen, Tafelbildern, Referaten oder schriftlichen Ausarbeitungen festgehalten werden.

\section{Individuelle Förderung}

Da die Teilnehmenden sich sowohl hinsichtlich ihrer schulischen Vorbildung als auch ihrer kognitiven Möglichkeiten und der persönlichen Lebenssituation stark unterscheiden, erstellen wir individuelle Förderpläne. In jedem Semester führt die Lehrgangsleitung Einzelgespräche, die durch einen Fragebogen geleitet werden. Hier werden die Eigen- und Fremdeinschätzungen der fachlichen und sozialen Kompetenzen festgehalten. Die Fremdeinschätzungen werden durch die Fachlehrerinnen und -lehrer ergänzt. In einer individuellen Zielvereinbarung wird jeweils das nächste Ziel, beispielsweise das Bestehen einer Prüfung, und die dazu erforderlichen Handlungsschritte festgehalten. Beim nächsten Gespräch können wir dann gemeinsam überprüfen, ob das Ziel erreicht wurde und gegebenenfalls durch gezielte Unterstützung einen Beitrag dazu leisten, dass dieses Ziel zu einem späteren Zeitpunkt erreicht werden kann. Wenn sich beispielsweise jemand häufig verspätet und so Unterrichtsinhalte versäumt und abgemahnt werden muss, so überlegen wir im Förderplangespräch gemeinsam, welche Ursachen die Verspätungen haben und wie man diese abstellen kann. In der Zielvereinbarung verpflichtet sich der Teilnehmende, künftig pünktlich zum Unterricht zu erscheinen. Hält er sich nicht an diese Vereinbarung, so kann dies nach zwei Abmahnungen zum Ausschluss aus dem Kurs führen. 
Oft sind auch individuelle Probleme wie Konzentrationsstörungen Inhalt des Gespräches. Hier versuchen wir eine gemeinsame Strategie zu entwickeln. Zunächst müssen wir herausfinden, wann diese Störungen auftreten. So hat z.B. ein Teilnehmer Schwierigkeiten, sich beim Lesen auf den Text zu konzentrieren. Im Gespräch fanden wir heraus, dass er Sachtexte als Einheit nicht gut überblicken konnte. Er konnte sich diese Texte jedoch mit Hilfe von Leitfragen schrittweise erarbeiten. Die Lehrerin bot an, ihm zunächst für solche Texte Leitfragen zur Verfügung zu stellen und er erklärte sich bereit, das Lesen auf diese Weise zu trainieren und zu versuchen, seine Frustration zu überwinden.

Fortschritte, Entwicklungen und Schwierigkeiten aller Teilnehmenden werden in den individuellen Förderplänen festgehalten und zur Evaluation eingesetzt.

\section{Eigenverantwortliches Handeln}

In den o.g Förderplänen definieren die Teilnehmenden selbst ihre Ziele für den jeweiligen Abschnitt des Lehrgangs. Sie übernehmen damit die Verantwortung für ihr Handeln im Lehrgang. Alle Teilnehmenden im Kurs erhalten Unterstützung außerhalb der Schule, um ihre persönlichen Angelegenheiten zu regeln. Das Ziel solcher unterstützender Maßnahmen ist die allmähliche Verselbständigung. Insofern ist das eigenverantwortliche Lernen in der Schule ein wichtiger Baustein, damit die Teilnehmenden künftig die Verantwortung für ihr Leben selbst tragen können. Wenn die jungen Erwachsenen im Lehrgang ihre Ziele selbst definieren, setzt dies voraus, dass sie sich mit ihrem aktuellen Leistungsstand und ihrer Lebenssituation intensiv beschäftigen und sich selbst einschätzen können. Dies ist eine wichtige Kompetenz für Menschen, die aufgrund einer psychischen Erkrankung lernen müssen, sich nicht zu überfordern und ihre Arbeit entsprechend einzuteilen. Viele Teilnehmende haben sehr hohe Ansprüche an sich selbst und setzen sich damit unter Druck, was dann häufig zum Scheitern führt, weil der Druck zu groß wird und Versagensängste hervorruft, die dann lähmend wirken und effektives Arbeiten verhindern. Es entsteht ein Teufelskreis, der unweigerlich dazu führt, dass die jungen Menschen wieder Misserfolge erleben und entmutigt werden, was dann das ohnehin negative Selbstbild bestätigt.

Ein Teilnehmer konnte sich im Unterricht zunächst nur mit Mühe konzentrieren. Er war neu in die bestehende Gruppe aufgenommen worden und fühlte sich sehr unsicher. Er war mißtrauisch und hatte oft das Gefühl, dass andere über ihn sprachen oder ihn nicht mochten. Gleichzeitig erschwerte ihm die Einnahme hochdosierter Medikamente zusätzlich die Konzentration. Er wirkte häufig überfordert und zeitweilig völlig abwesend. Obwohl im Lehrgang individuelle Pausen eingelegt werden können, bat er nie um eine Pause. Er wurde in schwierigen Situationen sehr still, raufte sich die Haare und wirkte verzweifelt, insbesondere wenn er Texte lesen musste. In Gesprächen mit der Lehrerin stelle sich heraus, dass er sich stark überfordert fühlte. Er konnte kaum einen Absatz eines Textes konzentriert lesen und den Inhalt überblicken. In Klassenarbeiten konnte er nur selten alle Aufgaben bearbeiten. Es stellte sich heraus, dass er sich in solchen Situationen stark unter Druck setzte, was wiederum Versagensängste hervorrief. Er war dann auch nicht dazu in der Lage, um eine Pause zu bitten. Im Förderplangespräch wurde vereinbart, dass der Teilnehmer eine Pause einlegen solle, wenn er sich überfordert fühle. Die Lehrerin wollte ihn unterstützen, indem sie ihm zunächst die Pause anbot, wenn er offensichtlich überfordert wirkte. 
15 Schule für Betreuung und berufliche Weiterbildung Jugendlicher und junger Erwachsener mit einer schizophrenen Erkrankung

\begin{abstract}
Weiterhin überlegte der Teilnehmer, welchen Anforderungen er in der aktuellen Situation gerecht werden konnte. Es wurde vereinbart, dass er sich für den aktuellen Lehrgangsabschnitt auf zwei Fächer konzentrieren wolle, die er inklusive aller Klassenarbeiten absolvieren würde. Den Unterricht in den anderen Fächern werde er regelmäßig besuchen, aber die Klassenarbeiten nicht schreiben. Dieses Pensum sollte allmählich gesteigert werden. Der Teilnehmer wirkte deutlich entlastet, obwohl es ihm schwer fiel, seine Ansprüche zu reduzieren. Er wollte noch immer das volle Pensum schaffen und musste sehr viel Selbstdisziplin aufbringen, um bei der Vereinbarung zu bleiben. Letztlich absolvierte er das Semester in mehr als zwei Fächern erfolgreich und konnte im folgenden Semester bereits Klassenarbeiten in allen Fächern schreiben. Durch das kontinuierliche kognitive Training in der Schule gelang es ihm, seine Konzentrationsfähigkeit zu verbessern und schließlich auch längere Texte bearbeiten zu können. Er entschloss sich, einen Lehrgangsabschnitt zu wiederholen, um die Anforderungen allmählich steigern zu können. Nach drei Jahren erreichte er auf diese Weise die Mittlere Reife mit Qualifikation (alle Noten waren mindestens befriedigend). Sowohl seine Leistungen als auch sein Selbstbild hatten sich deutlich verbessert, was in den Auswertungsbögen der Förderplangespräche deutlich erkennbar ist. Er wirkte entspannt und sicherer und hatte Kontakt zu allen Teilnehmern im Kurs.
\end{abstract}

Neben den Qualifikationen erwarb der Teilnehmer die Kompetenz, sein eigenes Handeln zu planen und zu evaluieren. Er hat gelernt, eigene Ansprüche zu kontrollieren und sein Selbstwahrnehmung verbessert. Dies ist eine wichtige Voraussetzung, damit er in der Folge Angebote außerhalb eines geschützten Rahmens wahrnehmen kann.

\title{
Psychologische Begleitung
}

Ein Psychologe und Verhaltenstherapeut steht dem gesamten Team beratend zur Seite. Er nimmt an den wöchentlichen Teamsitzungen teil und trägt dazu bei, Schwierigkeiten im Kurs zu meistern, die durch die Symptome der Teilnehmenden bedingt sind. Das Team erarbeitet dann Lösungen. Zugleich steht der Psychologe auch den Teilnehmenden zu Verfügung. Er unterricht zwei Wochenstunden „Methodentraining“, wo es neben der Psychoedukation darum geht, Konflikte in der Cruppe oder die Überforderung Einzelner schnell zu erkennen, anzusprechen und gemeinsame Lösungen zu erarbeiten. Dies ist besonders wichtig, weil der Alltag in der Lerngruppe für die jungen Menschen eine große Herausforderung darstellt. Viele Eindrücke müssen verarbeitet werden, einige Teilnehmende sind sehr skeptisch und misstrauisch gegenüber anderen. Sie fühlen sich schnell ausgegrenzt, beobachtet oder schlecht behandelt. Solche Gefühle müssen wir frühzeitig erkennen, um entgegenwirken zu können. Gelingt dies nicht, so kann für Betroffene der Schulbesuch sehr rasch unerträglich werden. Sie fühlen sich ständig unwohl, was zur Folge hat, dass sie dem Unterricht nicht mehr folgen.

\section{Fachunterricht und Berufsorientierung}

Der Unterricht wird gemäß $₫ 6$ des Weiterbildungsgesetzes in den Fächern Englisch, Mathematik, Deutsch, Geschichte, Biologie und Wirtschaftslehre erteilt. Der Projektunterricht ergänzt die Kernfächer. Das Fach Methodentraining ist eine Besonderheit des Kurses. Es wird von einem erfahrenen Psychologen und Verhaltenstherapeu- 
ten unterrichtet. Hier werden therapeutische Ansätze in den Unterricht integriert. Probleme und Konflikte in der Gruppe können angesprochen und Lösungsansätze entwickelt werden.

Zusätzlich werden die Teilnehmenden durch ein Begleitprojekt zur beruflichen Orientierung auf den Übergang in das Berufsleben vorbereitet. Bisherige Erfahrungen im Schulprojekt mit psychisch erkrankten jungen Erwachsenen an der VHS Essen zeigen, dass diese Cruppe besondere Förderkonzepte zur Vorbereitung auf das Berufsleben benötigt. Durch die oft langjährige Erkrankung haben sie meist nur noch sehr wenig Selbstvertrauen, und sie müssen im Alltag häufig Einschränkungen wie Ängste, Stimmungsschwankungen oder Konzentrationsstörungen überwinden. Gleichzeitig werden sie mit Stigmatisierungen durch die Allgemeinheit konfrontiert, die dazu führt, dass man ihnen mit Vorbehalten begegnet. Es wird unter diesen Voraussetzungen sehr schwer für sie sein, das Vertrauen potentieller Arbeitgeber zu gewinnen und den Einstieg in das Erwerbsleben zu schaffen. Sie brauchen aber Selbstvertrauen, Mut und kommunikative Kompetenz, um in der Arbeitswelt ihre besonderen Stärken darstellen und ihre schwierige Biographie überwinden zu können.

Im Rahmen des Projektes wird versucht, den jungen Erwachsenen durch Bewerbungstrainings, das Erlernen von Präsentationstechniken sowie das Erstellen von aussagekräftigen Bewerbungsunterlagen den Weg für eine erfolgreiche Bewerbung zu ebnen. Weiterhin werden sie durch theater- und kunstpädagogische Projekte gefördert, um Mut und Selbstvertrauen für das Leben außerhalb eines geschützten Rahmens aufzubauen. Für die psychologische Beratung und die psychosoziale Begleitung stehen den Teilnehmenden und Lehrenden ein Psychologe und die Projektleitung zur Verfügung. In das Projekt sind Betriebspraktika eingebunden, die eine berufliche Orientierung der Teilnehmenden fördern und sie allmählich auf den Arbeitsalltag vorbereiten.

\section{Teamarbeit und Vernetzung}

Die Arbeit mit jungen Menschen, die psychisch erkrankt sind, verlangt von den Lehrerinnen und Lehrern einen besonders sensiblen Umgang mit der Situation jedes einzelnen Teilnehmers. Sie müssen flexibel auf die individuellen Bedürfnisse eingehen und Überforderung frühzeitig erkennen und vermeiden. Die Lehrerinnen und Lehrer müssen ein vertrauensvolles aber abgrenzendes Verhältnis zu den Teilnehmenden aufbauen. Die pädagogischen Prozesse werden durch die Lehrgangsleitung moderiert.

Zum pädagogischen Team gehören neben der Lehrgangsleitung vier Fachlehrkräfte. Alle Lehrerinnen und Lehrer bringen neben der Fachkompetenz eine große Bereitschaft mit, sich auf die speziellen Bedürfnisse der Teilnehmenden einzustellen, sich weiterzubilden und mit großer Sensibilität Probleme und Krisen zu erkennen und zu deren Bewältigung beizutragen.

Das gesamte pädagogische Team arbeitet sehr eng zusammen und tauscht sich bei wöchentlichen Teamsitzungen regelmäßig aus. Diese Teamsitzungen werden fachlich durch den psychologischen Psychotherapeuten begleitet.

$\mathrm{Zu}$ den Teamsitzungen werden auch regelmäßig Teilnehmende eingeladen, insbesondere dann, wenn längere Fehlzeiten oder soziale Probleme in der Cruppe auftre- 
15 Schule für Betreuung und berufliche Weiterbildung Jugendlicher und junger Erwachsener mit einer schizophrenen Erkrankung

ten. Das folgende Beispiel veranschaulicht das besondere pädagogische Potential eines koordinierten Vorgehens im Kollegium.

\begin{abstract}
Über längere Zeit waren die Fehlzeiten im Lehrgang stark angestiegen und gleichzeitig zogen sich einige Teilnehmende sehr zurück und beteiligten sich kaum noch am Unterricht, falls sie überhaupt kamen. In dieser Phase wirkte ein Teilnehmer sehr dominant, der sehr leistungsstark war und sich durch den Unterrichtsstoff häufig unterfordert fühlte. Obwohl er zusätzliche und weiterführende Aufgaben erhielt, äußerte er häufig, dass alles „kein Problem" sei und man das Pensum im Lehrgang ohne Probleme "locker" schaffen könne. Wurden Aufgaben gestellt, so hatte er nach kurzer Zeit eine Lösung gefunden und verkündete lautstark, dass er nun fertig sei. Er wurde ungeduldig und störte den Unterricht. Andere Teilnehmende, die beim Lesen von Texten krankheitsbedingt Schwierigkeiten haben und mehr Zeit zur Bearbeitung benötigen, fühlten sich entmutigt und frustriert. Die überheblichen Reaktionen des unterforderten Kursteilnehmers wirkten auf andere Gruppenmitglieder herablassend und bestätigten deren Selbstzweifel. Sie mussten im Unterricht immer wieder ermutigt werden, sich zu beteiligen und die Aufgaben zu bearbeiten.
\end{abstract}

Das Team beschloss, den dominierenden Teilnehmer zur Teamsitzung einzuladen. In dem Gespräch wurden zunächst die gute Mitarbeit und die hohe fachliche Kompetenz des jungen Mannes gewürdigt. Im Anschluss wurde problematisiert, dass er andere Teilnehmende, durch sein Verhalten entmutigen und frustrieren könne, ohne dies zu beabsichtigen. Das Team bat inn darum, künftig auf solche Äußerungen zu verzichten, die andere Teilnehmende als abwertend empfinden könnten. Der Teilnehmer nahm das Lob für die gute Mitarbeit und seine Fachkompetenz mit Freude entgegen und konnte verstehen, dass sein Verhalten andere Teilnehmende entmutigte, die mit dem Lernstoff kämpften. Tatsächlich gelang es ihm, in der Folgezeit weniger dominant in der Gruppe aufzutreten. Die anderen Teilnehmenden kamen wieder regelmäßiger zum Unterricht und beteiligten sich an Unterrichtsgesprächen. In diesem Prozess entwickelte der „dominant“ wirkende Teilnehmer sein Sozialverhalten weiter, denn es gelang ihm mit Hilfe des Teams, auf andere Rücksicht zu nehmen und sie damit zu unterstützen.

Auch wenn Teilnehmende häufig fehlen oder überfordert wirken, hat es sich bewährt, sie zur Teamsitzung einzuladen. Als die ersten Einladungen ausgesprochen wurden, empfanden die Teilnehmenden es eher als unangenehm, vor dem Kollegium über ihre persönlichen Angelegenheiten sprechen zu müssen. Allerdings sprach sich schnell herum, dass es in diesen Gesprächen weniger um eine Disziplinierung, sondern vielmehr darum geht, gemeinsam eine Lösung für Probleme zu finden. Das Kollegium bietet seine Unterstützung an. Die Einladung zum Teamgespräch bietet den Lehrerinnen und Lehrern den Vorteil, dass sie gemeinsam ihr Anliegen verdeutlichen und ihm so Nachdruck verleihen können. Für die Teilnehmenden ist das Teamgespräch eine Möglichkeit, mit Unterstützung aller Lehrkräfte ein individuelles Problem oder Anliegen zu besprechen. Es ist auch ein Ausdruck von Wertschätzung, dass sich das gesamte Kollegium Zeit für einen einzelnen Teilnehmenden nimmt.

Auch die Betreuerinnen und Betreuer in den Einrichtungen der Professor Dr. Christian Eggers Stiftung und anderer betreuender Einrichtungen werden durch regelmäßige Gespräche in die Arbeit einbezogen. Leben die Teilnehmenden noch bei ihren Eltern, so können diese ebenfalls einbezogen werden. Hier ist ein sehr fruchtbarer 
Austausch entstanden, der bei der Bewältigung von Krisen und Problemsituationen im Kurs sehr hilfreich ist.

\subsection{Bisherige Resultate aus dem Modellprojekt}

Aus den Erfahrungen im Professor Eggers Lehrgang haben sich folgende Kernpunkte ergeben, die neben individuellen Strategien für einen erfolgreichen Schulbesuch besonders wichtig sind:

\section{Strategien für einen erfolgreichen Schulbesuch}

- Lehrerinnen und Lehrer begegnen den Teilnehmenden mit Wertschätzung und Respekt.

- Innerhalb der Cruppe besteht ein vertrauensvolles und offenes Verhältnis.

- Konflikte werden frühzeitig erkannt und Lösungen gemeinsam mit allen Beteiligten erarbeitet.

- Die Zahl der Unterrichtsstunden kann bei Bedarf zeitweilig und individuell reduziert werden.

- Lehrende und Teilnehmende achten darauf, dass sie Überforderung rechtzeitig bemerken oder noch besser: vermeiden.

- Die Teilnehmenden bauen Vertrauen in die eigenen Fähigkeiten auf und übernehmen die Verantwortung für ihr Handeln.

- Übergangsschritte in Angebote außerhalb eines geschützten Rahmens werden sorgfältig geplant und Anforderungen allmählich gesteigert.

Nach den ersten vier Jahren lässt sich zusammenfassend feststellen, dass psychotisch oder anderweitig psychisch erkrankte junge Erwachsene durch gezielte Förderung und kognitives Training im Professor-Eggers-Lehrgang einen qualifizierten Schulabschluss erreichen können. Tabelle 88 dokumentiert, wie viele Schulabschlüsse erreicht werden konnten und mit welcher schulischen Vorbildung die Teilnehmenden aufgenommen wurden.

Im ersten Durchgang des Lehrgangs erreichten 7 von 10 Teilnehmenden, im zweiten Durchgang 14 von 15 Teilnehmende des vierten Semesters den mittleren Bildungsabschluss, davon eine Teilnehmerin in einer Einzelfachprüfung in Englisch. Viele Teilnehmende nahmen an den Prüfungen zum Hauptschulabschluss nach Klasse 9 oder 10A ebenfalls teil, so dass einige im Kurs bis zu drei Abschlüsse stufenweise erwarben. Diese Erfolge führten auch dazu, dass der Lehrgang inzwischen nicht mehr aus Spendenmitteln finanziert werden muss, sondern sich über die Eingliederungshilfe für die einzelnen Teilnehmenden finanziert, die von den Jugend- und Sozialämtern der Stadt Essen und umliegender Kommunen auf Antrag gewährt werden kann.

Sieben ehemalige Absolventinnen und Absolventen besuchen heute das Abendgymnasium oder ein Berufskolleg und bereiten sich auf ihr Fachabitur vor. Sie hatten alle entweder gar keinen oder einen wenig zufriedenstellenden Schulabschluss, als sie in den Professor-Eggers-Lehrgang aufgenommen wurden. Ihre Hoffnung, jemals einen qualifizierten Schulabschluss erreichen zu können, war sehr gering. Die Mutter eines Teilnehmers berichtete, dass man ihr gesagt habe, ihr Sohn werde niemals einen Schulabschluss erreichen können. Tatsächlich wirkte er im Lehrgang zunächst 
15 Schule für Betreuung und berufliche Weiterbildung Jugendlicher und junger Erwachsener mit einer schizophrenen Erkrankung

Tab. 88 Übersicht im Professor-Eggers-Lehrgang erworbene Abschlüsse (2006-2010)

\begin{tabular}{|c|c|c|c|c|}
\hline Teilnehmer & $\begin{array}{c}\text { Abschluss } \\
\text { vor Aufnahme }\end{array}$ & HSA 9 & HSA 10A & MBA \\
\hline TN1 & kein Abschluss & $x$ & $x$ & $x$ \\
\hline TN2 & kein Abschluss & $x$ & $x$ & $x$ \\
\hline TN3 & kein Abschluss & & $x$ & $x$ \\
\hline TN4 & kein Abschluss & $x$ & $x$ & $x$ \\
\hline TN5 & kein Abschluss & $x$ & $x$ & $x$ \\
\hline TN6 & kein Abschluss & $x$ & $x$ & $x$ \\
\hline TN7 & kein Abschluss & & $x$ & $x$ \\
\hline TN8 & kein Abschluss & $x$ & $x$ & \\
\hline TN9 & HSA 9 & & $x$ & $x$ \\
\hline TN10 & HSA 9 & & & $x$ \\
\hline TN11 & HSA 9 & & & $x$ \\
\hline TN12 & HSA 9 & & & $x$ \\
\hline TN13 & HSA 9 & & $\mathrm{x}$ & $x$ \\
\hline TN14 & HSA 9 & & $x$ & $x$ \\
\hline TN15 & HSA 9 & $\mathrm{x}$ & $x$ & $x$ \\
\hline TN16 & HSA 9 & & $x$ & $x$ \\
\hline TN17 & HSA 9 & & & \\
\hline TN 18 & HSA 9 & & & Einzelfach Englisch \\
\hline TN19 & HSA 10A & & $x$ & $x$ \\
\hline TN20 & HSA $10 \mathrm{~A}$ & & & $x$ \\
\hline TN21 & HSA $10 \mathrm{~A}$ & & & $x$ \\
\hline TN22 & HSA $10 \mathrm{~A}$ & & $x$ & $x$ \\
\hline TN23 & HSA $10 \mathrm{~A}$ & & & $x$ \\
\hline TN24 & HSA $10 \mathrm{~A}$ & & & \\
\hline TN25 & HSA $10 \mathrm{~A}$ & & & \\
\hline \multicolumn{5}{|c|}{ HSA 9: Hauptschulabschluss nach Klasse 9} \\
\hline \multicolumn{5}{|c|}{ HSA10: Hauptschulabschluss nach Klasse $10 \mathrm{~A}$} \\
\hline \multicolumn{5}{|c|}{ MBA: mittlerer Bildungsabschluss (Fachoberschulreife) } \\
\hline
\end{tabular}

sehr abwesend und oft nicht ansprechbar. Er hörte Stimmen und war deshalb sehr abgelenkt und benötigte immer wieder Pausen während der Unterrichtsstunden. Allerdings verbesserten sich sowohl seine Konzentrationsfähigkeit, als auch seine Leistungen während des Lehrgangs stetig. Er erwarb einen mittleren Bildungsabschluss mit guten Noten und bereitet sich nun am Berufskolleg auf das Fachabitur vor und denkt über ein späteres Studium nach. Dieses Beispiel zeigt, dass Prognosen oft vorschnell gestellt werden und den Betroffenen eher schaden als nützen. Es ist 
entmutigend und demotivierend, einem jungen Menschen jede Hoffnung zu nehmen, ein Ziel zu einem späteren Zeitpunkt vielleicht doch noch erreichen zu können. Solche Prognosen fördern ein passives Verhalten. Letztlich sind sie sicher ein Faktor, der die Chronifizierung von Erkrankungen befördert. Wir erleben täglich, dass auch junge Erwachsene, die Stimmen hören oder dissoziieren, die zeitweilig nur an wenigen Unterrichtstunden teilnehmen können und gegen die starken Nebenwirkungen ihrer Medikamente ankämpfen müssen, trotz ungünstiger Prognosen und mit gezielter, wohlwollender Unterstützung ihr Ziel erreichen. 